\title{
Learning a unimanual motor skill by partial commissurotomy patients
}

\author{
Y P Chen, R Campbell, J C Marshall, D W Zaidel
}

\begin{abstract}
A series of motor tests on four Chinese partial commissurotomy patients is reported. The single-stage commissurotomy in all four patients included the anterior commissures and twothirds or four-fifths section of the corpus callosum with sparing of the splenium. There was no demonstrable ability to transfer hand posture in these patients. This was the major evidence for functional deconnexion. A newly learned task of one-hand knotting revealed right hand impairment in all four patients. There was no dyspraxia in the right hand for over-learned object-handling tasks in these patients. It is suggested that there might be right hemisphere specialisation for the initial acquisition of unimanual object-handling skills and that the spared callosal fibres in the splenium alone are insufficient to mediate task control under these conditions. This is supported by the finding that one of these patients, who was the only one who had a right parietal lesion, was unable to perform the newly learned task with either hand.
\end{abstract}

The role of the callosal fibres in controlling the motor performance of the left hand was first emphasised by Liepmann ${ }^{1-4}$ in his attempt to explain left hand apraxia in the presence of right hemiplegia. These early discussions attributed a greater role to the left than the right hemisphere in volitional control of motor activity in both the right and left hands. Later, this notion was supported by observations that left hemisphere damage may result in ideomotor apraxia in both hands ${ }^{5}$ or in impairments of various motor functions such as rapid target-directed movements, ${ }^{6}$ those involved in rotary pursuit, ${ }^{7}$ in copying meaningless sequences of hand and arm movements, ${ }^{8}$ and in acquiring a meaningless unimanual motor skill. ${ }^{6}$ It has also been shown that in patients who had only a partial section of the corpus callosum with sparing of the splenium, control of newly learned motor skills is impaired in tasks requiring interdependent bimanual movements. ${ }^{910}$ This suggests a crucial role of the forebrain commissures in such motor tasks. ${ }^{9-10}$

Callosal fibres are important in mediating unilateral motor control for which there is hemispheric specialisation and in mediating certain aspects of bimanual movements in newly learned motor tasks. ${ }^{9-10}$ It is therefore reasonable to ask what role, if any, they play in the initial learning of a new unimanual skill involving object-handling. This has not been studied previously. Thus in our study, the focus is on determining the extent of lateralised motor control in unimanual object-handling by partial commissurotomy patients in Shanghai, China. The patients are comparable to the Caltech series ${ }^{9-10}$ as they had anterior section of the callosum as well as a complete section of the anterior commissure.

The guiding principle when designing the new task was that it should be ecologically valid. It consists of a series of sequential movements which are mutually interdependent, all leading to a goal, namely, a string knot. In this sense it is rendered familiar in terms of everyday life. However, the four mutually interdependent steps as well as its unimanual aspect are new, and must be acquired by learning. In view of previous evidence in support of left hemisphere specialisation in learning sequential movements, ${ }^{6}$ this skill may favour the left hemisphere. An opposing view is that since it emphasises spatial features of motor output it may favour the right. Thus initially, no prediction regarding the specific pattern of results was made.

\section{Method}

Subjects

The tests were carried out on four righthanded Chinese patients (one female and three males) in the Institute of Neurology in Huashan Hospital attached to Shanghai Medical University. They all had single-stage, partial surgical section of the corpus callosum by retraction of their right hemispheres for the relief of intractable epilepsy: in one (SM1), the anterior four-fifths of the callosum was cut whereas in the remaining patients the anterior two-thirds was cut. The anterior commissure was divided in all patients. Table 1 summarises the case histories and shows the location of extra-callosal damage as verified by CT. After surgery, seizures were reduced in all four patients. The normal control subjects were four right-handed non-brain damaged inpatients in the same neurology department of Huashan Hospitals. They were matched for age, sex, education, and socio-economic level, with the four commissurotomy patients.

The tests we describe were given after routine neurological examination revealed normal proprioception and strength in either 
Table 1 Summary of Case Histories

\begin{tabular}{lllll}
\hline Name & $S M 1$ & $S F 2$ & $S M 3$ & $S M 4$ \\
\hline Handness & $\mathbf{R}$ & $\mathbf{R}$ & $\mathrm{R}$ & $\mathbf{R}$ \\
Sex & $\mathrm{M}$ & $\mathbf{F}$ & $\mathbf{M}$ & $\mathbf{3}$ \\
Education (Years) & 2 & 4 & Housework & Housework \\
Current occupation & Housework & Janitor & Encephalitis & Absence \\
History of brain damage & Head injury & Absence & R-Frontal & R-Parietal \\
Extracallosal damage (CT Scan) & R-Frontal & Absence & 19 & 20 \\
Age at surgery (years) & 14 & 30 & 21 & 22 \\
Age at testing (years) & 16 & 32 & 5 & 10 \\
Age at first seizure (years) & 7 & 13 & & \\
\hline
\end{tabular}

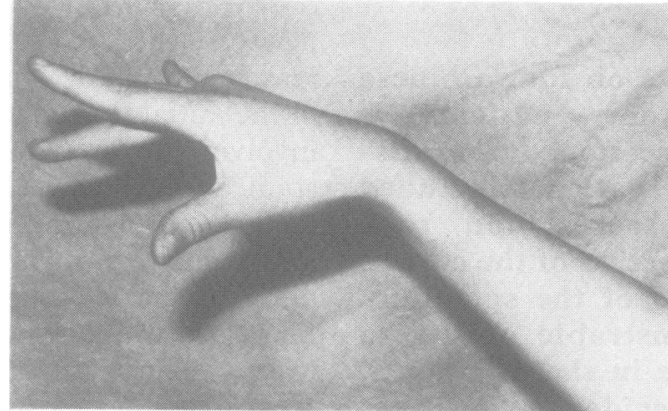

Figure 1 Imitating a single hand posture.

hand. They were administered two years after surgery. No verbal instructions were provided by the examiner on how to perform these tasks and no time limit was imposed. Instead, patients were asked to learn what was required through watching and imitating the examiner. In these unimanual tests, each hand was always examined separately with the left hand tested first.

On all of the tests, the four non-brain damaged control subjects performed flawlessly.

1a) Imitating a hand posture With the patient's eyes closed, the examiner imposed a hand posture on one hand and the subject's task was to duplicate it with the other. The testing order of a hand-posture model to a hand-posture copy was counterbalanced between the two hands and repeated at least three times before patients gave up their attempts. Only one simple hand posture was used (fig 1). None of the patients was able to carry out this task, suggesting a failure to cross-transfer proprioceptive manual information between the two hemispheres. But when, in free-vision, the patient was asked to mimic above, performance was normal in all patients. b) Imitating hand action The examiner used her thumb to touch each finger in turn on one hand. The patients were asked to repeat the same sequence on their own fingers. Five different sequences were administered for each hand and the results indicated normal performances by all four patients with either hand.

2 Over-learned object-handling skill This was tested by asking patients to demonstrate the unimanual use of everyday objects: chopsticks for picking up at least three of five blocks displayed in free vision, scissors for cutting an index card with one end fixed to a board and string fixed at one end for free knotting (fig 2). No deficits were found in three of the the examiner who used the same posture as patients, but while patient SM4 used these three objects flawlessly in his right hand, he showed impairment in his left. This was especially marked when using chopsticks and knotting string. Some improvement was noted when the examiner placed the objects in the proper spatial relationships between his fingers. However, no improvement was observed in his awkward left hand performance when the examiner demonstrated the correct use or when verbal guidance was provided (such as "put your middle finger between the two chopsticks").

3 Unimanual knotting learning (UKL)

The examiner used a piece of string attached to a board to demonstrate how to tie a knot in a new way. Demonstrations were given until each patient announced their understanding of the task. The procedure for tying the knot was then demonstrated in four discrete steps (fig 3) with the examiner sitting next to the patient rather than facing the patient. Patients were first asked to repeat the required hand posture immediately after each step was demonstrated. At this point the examiner was holding the string but the patient was not. required to make the knot on their own using the four sequential steps. Patients were encouraged to try at least three times before giving up. They were asked to perform this task first with the left hand and then with the right hand. Before the right hand trials, the four steps were again demonstrated by the examiner.

\section{Results}

The results revealed no impairment in repeating the correct hand posture for each of the four steps required for making the knot. The impairments that were noted related to the

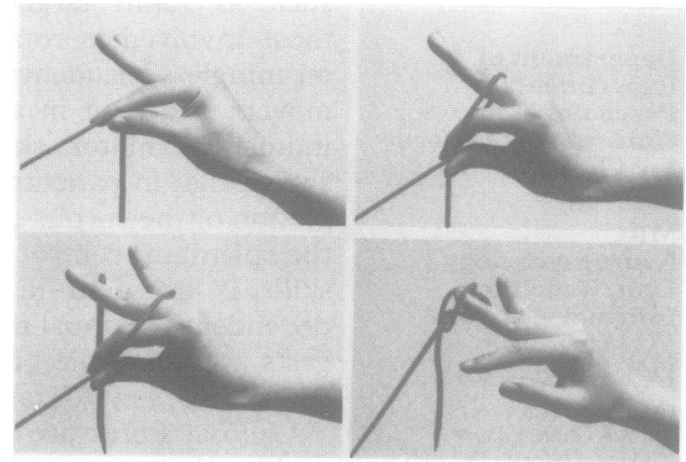
Then, with the string in their hand, they were

Figure 3 Four steps in learning a new unimanual task: tying a knot in a new way (left to right and top to bottom tying a knot, cutting with scissors, using chopsticks to pick up a block. 


\begin{tabular}{|c|c|c|c|c|c|c|c|c|}
\hline & \multicolumn{4}{|c|}{ Left hand } & \multicolumn{4}{|c|}{ Right hand } \\
\hline & SM1 & $S F 2$ & SM3 & SM4 & SM1 & $S F 2$ & SM3 & SM4 \\
\hline \multicolumn{9}{|l|}{ 1a Imitation of Hand posture } \\
\hline Blind & + & + & + & + & + & + & + & + \\
\hline Sighted & - & - & - & - & - & - & - & - \\
\hline 1b Imitation of Hand action & - & - & - & - & - & - & - & - \\
\hline \multicolumn{9}{|l|}{2 Over-learned Object-handling } \\
\hline Chopsticks & - & - & - & + & - & - & - & - \\
\hline Scissors & - & - & - & + & - & - & - & - \\
\hline Free Knotting & - & - & - & + & - & - & - & - \\
\hline \multicolumn{9}{|l|}{3 UKL } \\
\hline a Imitation of Each posture & - & - & - & - & - & - & - & - \\
\hline b String-knotting in a novel way & - & - & - & + & + & + & + & + \\
\hline
\end{tabular}

knotting task and they were as follows: three patients (SM1, SF2, SM3) tied the knot with the left hand normally but had right hand impairment. They appeared unable to orient and place the string properly with their fingers and finally failed to make a knot in this new way with their right hands. Spontaneously, they expressed astonishment at their right hand deficit since none believed that the nonpreferred left hand would be more dextrous by comparison.

Performance by patient SM4 with a right parietal lesion showed bilateral hand impairment. The nature of his difficulty with the right hand was similar to that described above for the other patients. With the left hand he held the string awkwardly and perseverated (for example, he repeated producing a circle). See table 2.

\section{Discussion}

Tests for hand posture copying and action as well as over-learned and newly learned objecthandling unimanual tasks were administered to four partial commissurotomy patients. A functional disconnexion for cross-transfer of a simple finger posture imposed by the examiner was shown. In addition, three of the patients displayed right hand impairment, and one (SM4), who had a right parietal lesion, showed bilateral impairment in learning unimanual knotting. Except for the right parietal patient (SM4) who showed awkward performance in his left hand but not the right, all the other patients performed flawlessly in the over-learned object-handling task.

This result cannot be easily explained by the extra-callosal damage in these four patients, since none of them had CT evidence for extracallosal damage or possible damage by retraction during operation in the left hemisphere and all of them showed impaired performance in the right hand rather than the left. The result indicates that the acquisition of unimanual knotting draws predominantly upon the capacities of the right hemisphere, especially the right parietal lobe. In addition, they suggest that callosal mediation through fibres in the splenium is insufficient for the kind of control needed for a unimanual motor task, when a real object is used and a meaningful goal must be achieved. This notion is supported by the fact that the right parietal patient (SM4) had impaired performance in both hands for the unimanual knot learning task. He showed awkward performance in his left hand for both over-learned and newly learned objecthandling tasks; the right parietal lobe may therefore be essential for object-handling skills because this kind of skill places high demands on the tactuo-proprioceptive system and its coordination with motor output. The right parietal lobe appears to be crucial for establishing a perceptual-kinaesthetic motor programme for the acquisition of fine unimanual object-handling skill; both parietal lobes may be needed to programme motor output systems to carry out established objecthandling skills.

More generally, the present results suggest that unimanual hemispheric control may be determined by the nature of the newly learned motor task. Right hemisphere specialisation may be found if the unimanual motor task (such as UKL) demands complex coordination between the tactuo-proprioceptive and motor systems; if it involves many spatial features, and if it comprises an intrinsically ordered successsion of mutually interdependent steps that lead to a meaningful end-product. By contrast, left hemisphere specialisation is observed when a newly learned motor task involves discrete unimanual postural movements and the sequential steps are arbitrarily predetermined and neither mutually interdependent nor lead to one end-product. Kimura's sequential learning task ${ }^{6}$ which consists of pushing a button with the index finger, pulling a bar with four fingers, and then pressing a lever with the thumb, all in correct sequence, is a prime example of such a unimanual left hemisphere task.

These results with partial commissurotomy patients highlight two important features of hemispheric specialisation and motor control: 1) The right hemisphere may play an important role in unimanual motor control. When a spatial task is inherently sequential and as long as the subcomponents are interdependent and have a coherent meaningful context, the right hemisphere may be dominant for acquisition of the task. 2) The presence of spared callosal fibres in the splenium alone has been shown to be insufficient for the mediation of bimanual 
interdependent movements. ${ }^{910}$ This study suggests that the acquisition of a unimanual motor task cannot rely on spared splenium callosal fibres in tasks for which the right hemisphere is specialised.

Y P Chen is supported by the Sino-British Friendship Scholarship Scheme (SBFSS). D W Zaidel is supported by a Fulbright Fellowship and NIH NS 18973. We are grateful to Professor Zhen Qin and Dr Yu-xia Wan for providing detailed clinical reports on these cases.

1 Liepmann H. Das Krankheitsblid der (motorische Asymbolie) auf Grund eines Falles von einseitiger. Apraxie bolie) auf Grund eines Falles von einseitiger.
Monatasschr Psychiat Neurol 1900;8:182-97.

2 Liepmann H. Drei Aufstatze aus dem Apraxiegebeit. Berlin: Karger 1908.
3 Liepmann H, Mass O. Fall von linksseitiger Agraphie und Apraxie bei rechsseitiger Lahmung. J Psycho

4 Geschwind N. Disconnection syndrome in animal and man (II). Brain 1965;88:586-644

5 Hecaen $\mathrm{H}$. Clinical symptomatology in right and left hemisphere lesions. In: Mountcastle, VB ed. Interhemispheric relations and cerebral dominance, Baltimore: John Hopkins University Press, 1962:215-43.

$6 \mathrm{Kimura} \mathrm{D}$. Acquisition of a motor skill after left-hemisphere damage. Brain 1977;100:527-42.

7 Wyke $M$. Effect of brain lesions on the rapidity of arm movement. Neurology (Minneapolis) 1976;17:1113-20.

8 Kimura D, Archibald Y. Motor function of the left hemisphere. Brain 1974;97:337-50.

9 Zaidel D, Sperry RW. Some long-term motor effects of commissuorotomy in man. Neuropsychologia 1977;15: 193-204.

10 Preilowski BFB. Possible contribution of the anterior forebrain commissures to bilateral motor coordination. Neuropsychologia 1972;10:267-77. 